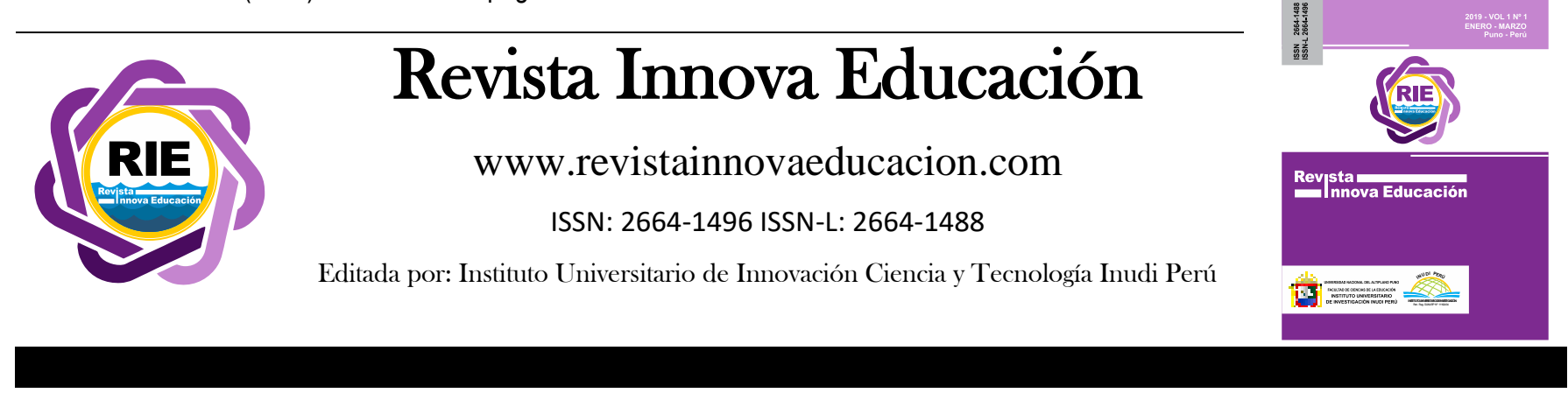

\title{
Diseño y validación del cuestionario "Actitud hacia la investigación en estudiantes universitarios"
}

\section{Design and validation of the questionnaire "Undergraduate students' attitude towards research"}

\author{
Erika Barrios ${ }^{1}$ \\ Universidad Autónoma del Estado de Morelos, Cuernavaca - Estado de Morelos, México \\ http://orcid.org/0000-0001-6214-0779 \\ Delgado Ulises ${ }^{2}$ \\ Universidad Autónoma del Estado de Morelos, Cuernavaca - Estado de Morelos, México \\ http://orcid.org/0000-0002-4318-0238
}

DOI: https://doi.org/10.35622/j.rie.2020.02.004

Recibido 17/03/2020 Aceptado 25/04/2020 Publicado 26/04/2020

ARTÍCULO ORIGINAL
PALABRAS CLAVE
actitud, cuestionario,
investigación,
universitarios,
validación

\section{KEYWORDS}

attitude, questionnaire, research, undergraduate students, validation

\begin{abstract}
Resumen: La actitud hacia la investigación es un tema a tomar en cuenta para quienes desean iniciar a los estudiantes universitarios en el mundo de la investigación, ya que los prejuicios y actitudes negativas pueden obstaculizar el aprendizaje. El objetivo de esta investigación fue diseñar y validar un cuestionario para evaluar la actitud hacia la investigación en estudiantes universitarios. A partir de la técnica de redes semánticas, se creó un cuestionario de 28 reactivos, que fue aplicado a 212 estudiantes de Psicología de 3 universidades. Se obtuvo un Alpha de Cronbach de .726 y en el análisis factorial resultaron 2 factores (actitud positiva y negativa) que explicaban el $30.24 \%$ de la varianza. Se encontró que los estudiantes de primer año tienden a tener mejor actitud hacia la investigación y esta va disminuyendo conforme avanzan en la carrera. En conclusión, el cuestionario mostró valores psicométricos aceptables para su uso.
\end{abstract}

Summary: Attitude towards research is a topic to consider for those who want to initiate undergraduate students into research, being that prejudices and negative attitudes can hinder learning. The objective of this research was to design and validate a questionnaire to assess the attitude of undergraduate students towards research. Using the semantic network technique, a 28 -item questionnaire was created, which was applied to 212 Psychology students from 3 different universities. A Cronbach's Alpha of .726 was obtained and, in the factor analysis, two factors (positive and negative attitude) were found, which explained $30.24 \%$ of the variance. It was established that freshman students tend to have a better attitude towards research which decreases as they advance in their degree. In conclusion, the questionnaire showed acceptable psychometric values for its use.

\footnotetext{
${ }^{1}$ Correspondencia: erika.barrios.gonzalez@gmail.com

${ }^{2}$ Correspondencia: ulises.delgado@uaem.mx
} 


\section{INTRODUCCIÓN}

En la sociedad del conocimiento las universidades deben impulsar un modelo de investigación, no solo entre sus docentes, sino en la formación de jóvenes investigadores, invirtiendo recursos humanos y materiales para lograr un modelo de investigación formativa de calidad (Canales, 2011).

Los beneficios de hacer investigación en pregrado están relacionados con el desarrollo del pensamiento crítico, lectura de comprensión y habilidades comunicativas, especialmente saber hablar y saber escribir con base en evidencias (García, Paca, Arista, Valdez \& Gómez, 2018). Es importante promover experiencias tempranas en investigación, ya que los estudiantes no sólo adquieren mayores conocimientos sobre el proceso, sino también confianza en sus habilidades y una identidad como generadores de conocimiento. Además, incrementan sus probabilidades de ingreso a estudios de posgrado al concluir su carrera (Adedokun, Zhang, Parker, Bessenbacher, Childress, \& Burgess, 2012).

Existen ciertas habilidades que parecen no modificarse con el sistema tradicional de enseñanza, esto se refleja en las investigaciones que comparan estudiante con y sin experiencia en investigación. En el estudio de Rocchi, Beaudry, Anderson y Pelletier (2016) compararon a estudiantes que habían tenido alguna experiencia en investigación con aquellos que no la tenían, encontrando diferencias con respecto al conocimiento y actitud. Aquellos que realizaron investigación consideraban que era una actividad placentera, mientras que aquellos que nunca han hecho investigación tenían una visión negativa, aparentemente debido a los prejuicios. Estos autores, señalan que es importante promover los beneficios de esta actividad entre los estudiantes, animándolos a participar en actividades extracurriculares de investigación. Otro estudio que hace una comparación similar es el de Barrios, Delgado y Hernández (2019) quienes encontraron que, aunque la experiencia en investigación no elimina por completo algunos prejuicios o errores en el proceso de investigación, aquellos que han tenido la experiencia de ejecutar un proyecto durante el pregrado, muestra una mejor disposición y una mejor actitud hacia esta actividad.

En el estudio de Taraban y Logue (2012) señalan que los beneficios que presentan los estudiantes mediante la práctica de la investigación, no se presentan en estudiantes que solo tienen estudios teóricos. Por su parte, Linn, Palmer, Baranger, Gerard, y Stone (2015) en su estudio sobre el impacto y oportunidades de estudiantes de pregrado en investigación, señalan igualmente, la importancia de tomar en cuenta las creencias y expectativas para el éxito de las experiencias en la formación de jóvenes investigadores.

El presente estudio se dirige a la exploración de la actitud hacia la investigación, reconociendo que "la actitud no es innata en el estudiante, depende de diversos aspectos, como la 
motivación intrínseca, las condiciones materiales y organizacionales de la institución, y en gran medida de los procesos de enseñanza-aprendizaje por el que haya transitado" (Ortega, Veloso \& Hansen, 2018, p. 108).

Los estudiantes al inicio de su carrera suelen tener una buena disposición a la investigación, pero los obstáculos y las malas experiencias van modificando esa actitud. Los estudiantes universitarios reconocen como obstáculos para realizar investigación, especialmente, la falta de tiempo y apoyo docente e institucional (Bendezú, Hurtado, Medina, \& Aguilar, 2015). En el estudio de Castro, Sihuay-Torres y Perez-Jiménez (2018) encontraron que los principales obstáculos que identifican los estudiantes universitarios para hacer investigación son: la falta de un asesoramiento adecuado y la falta de tiempo. Otro obstáculo que resalta en la literatura es la resistencia por la estadística, incluso para algunos estudiantes es una sorpresa que se requiera el uso de las matemáticas en las ciencias sociales o humanidades. Considerando las matemáticas como algo difícil que prefieren evitar. (Escalante, Repetto \& Mattinello, 2012; Pérez, Aparicio, Bazán \& Abdounur, 2015). Dependiendo el contexto pueden variar los factores que van modificando la disposición de los estudiantes universitarios a la investigación.

En el estudio de Chara-Saavedra y Olortegui-Luna (2018) se analizaron los factores asociados a una actitud desfavorable hacia la investigación, identificando que trabajar más de 10 horas a la semana, no haber recibido capacitación y leer solo un artículo a la semana, son identificados como factores de riesgo. Otro factor asociado a la actitud desfavorable son las expectativas, en el estudio de Adedokun y Burgess (2011) se analizaron las ideas que tenían los estudiantes con respecto a la investigación, encontrándose expectativas altas con respecto a la relación con sus mentores y al trabajo en equipo, se identificó que estas ideas podían afectar la experiencia que tenían. Estos autores señalan la importancia de trabajar las expectativas, ya que estas pueden afectar la experiencia y decisiones del estudiante. Sin embargo, no todos los estudios señalan una actitud negativa hacia la investigación, algunos estudios han encontrado valoraciones positivas de la actividad científica en estudiantes universitarios.

Es importante promover una actitud favorable hacia la investigación ya que esto puede aumentar el número de estudiantes que decidan titularse por tesis, una actividad que tendría efectos profundos en su formación (Mamani, 2015). Dentro de las valoraciones positivas, en el estudio de Ortega, Veloso y Hansen (2018) encontraron que el 90\% de los estudiantes consideran que la investigación contribuye en su formación profesional, y el 80\% les gustaría participar en un Semillero de Investigadores. De acuerdo con Cabrera-Enríquez et al (2013) "los factores asociados con una buena actitud fueron: pertenecer a un grupo de investigación, participar en más de dos 
proyectos de investigación, contar un asesor" (p.172), y ser mujer. La variable género es una variable que ha tenido resultados inconsistentes en los estudios, como se analizará más adelante.

Por su parte, Castro, Sihuay-Torres y Pérez-Jiménez (2018) encontraron que los estudiantes consideran que la investigación es importante porque aumenta el conocimiento científico, el prestigio y ayuda a obtener un mejor curriculum. Esta valoración no sólo debe provenir de los estudiantes, sino de las propias instituciones que, aunque tienen una desarrollada conciencia de la protección de datos y confidencialidad, existen pocos conocimientos respecto a la protección de la propiedad intelectual, más aún en la producción estudiantil (Acuña, Schmal \& Klein, 2011).

Los esfuerzos institucionales para promover la investigación suelen limitarse al curriculum con contenidos en investigación, sin embargo, Criollo, Romero y Fontaines-Ruiz (2017) señalan la importancia de visualizar la investigación como práctica transversal para que el estudiante no la vea como una materia más para aprobar, que impide un aprendizaje crítico y reflexivo.

Taraban y Logue (2012) mencionan la importancia de tomar en cuenta factores como las creencias y actitudes de los estudiantes, esto debido al impacto que tienen en las prácticas investigativas, por lo que a la hora de diseñar programas para promover la investigación en pregrado se deben considerar. Se deben tener instrumentos adecuados para evaluar estas predisposiciones a la investigación, sin embargo, Arellano-Sacramento, Hermoza-Moquillaza, Elías-Podestá y Ramírez-Julca (2017) señalan que existen pocos cuestionarios con respecto a la actitud hacia la investigación, y que los pocos que existen no son claros respecto al proceso de validación.

\section{1) Cuestionario de actitud hacia la investigación en universitarios}

En el cuestionario de Alonso, Alonso, y Valadez (2015) para evaluar la actitud hacia la investigación científica en estudiantes de enfermería, se obtuvo la validez de contenido por jueces y posteriormente un Alfa de Cronbach de 0.817. Las limitaciones que presenta este cuestionario es que no se brindan datos sobre la validez de constructo, esto puede estar relacionado al tamaño de la muestra que fue de solo 32 estudiantes.

En el caso del cuestionario de Aldana de Becerra, Caraballo, y Babativa (2016), también llamado Escala eacin, fue diseñado a partir de 3 dimensione: afectiva, cognitiva y conductual. Obtuvieron la validez de contenido a partir de jueceo y la confiabilidad con un Alfa de Cronbach de .854. Sin embargo, una característica del proceso de evaluación de propiedades psicométricas del cuestionario es que fue aplicado a alumnos (pregrado y posgrado), docentes y administrativos, 
ya que está dirigida a la comunidad académica, sin embargo, este resulta un grupo muy heterogéneo, puesto que son figuras académicas con motivaciones, ideas y antecedentes distintos.

En el estudio de Ochoa-Vigo, Bello, Villanueva, Ruiz-Garay y Manrique (2016) se obtuvo la validez de contenido a partir de 10 expertos, para la confiabilidad se obtuvo un Alfa de Cronbach de .680. El cuestionario estaba compuesto por dos dimensiones: percepción y actitud hacia la investigación. Arellano-Sacramento, Hermoza-Moquillaza, Elías-Podestá y Ramírez-Julca (2017) propusieron el diseño de un cuestionario de actitud hacia la investigación, dirigido especialmente a estudiantes de Estomatología. Obtuvieron un coeficiente Alfa de Cronbach de .650 y 6 factores que explicaban el $36.15 \%$ de la varianza, se requieren más estudios para aplicarlos en otras poblaciones. En el estudio de Castro (2017) se diseñó un cuestionario para evaluar la actitud hacia la investigación en estudiantes universitarios. Se evaluó la validez de contenido mediante un jueceo. Después de realizar un pilotaje se obtuvo la versión final del cuestionario que se organizó en 5 dimensiones (satisfacción-agrado, apropiación conceptual, comportamientos de aprendizaje, exploración sistemática y habilidades percibidas) que explican el 56,44 \% de la varianza. Para determinar la confiabilidad del cuestionario se obtuvo un Alfa de Cronbach de 0.827. En el estudio de Rey (2019) no se brindan datos de las propiedades psicométricas del cuestionario de actitud hacia la investigación utilizado, solo se señala que está compuesto por las dimensiones: afectiva, cognitiva y conductual.

Estos cuestionarios suelen seguir un proceso de construcción similar, en donde los investigadores construyen el cuestionario en función de otros cuestionarios o la revisión de la literatura, sin tomar en cuenta las ideas, prejuicios y creencias de sus propios estudiantes. Posteriormente un grupo de jueces validan el cuestionario, para después obtener sus propiedades psicométricas mediante la aplicación del cuestionario. El diseño del presente instrumento parte de la técnica de redes semánticas, que permite crear cuestionarios a partir de la representación sociales de los propios participantes, en combinación con lo que se encuentra en la literatura. Esta técnica ya ha sido utilizada en la construcción de cuestionarios como es el trabajo de Álvarez-Rebolledo, Santos, y Barrios (2019).

\section{2) Variables relacionadas con la actitud hacia la investigación}

Las variables más estudiadas en la literatura con respecto a la actitud hacia la investigación en estudiantes universitarios han sido: sexo, escuela y semestre, y aunque en algunos estudios no se han encontrado diferencias significativas (Arellano-Sacramento, Hermoza-Moquillaza, ElíasPodestá \& Ramírez-Julca, 2017; Chara-Saavedra \& Olortegui-Luna, 2018; Rey, 2019), en aquellos donde se muestran diferencias, no parece haber un consenso. 
Con respecto a la variable sexo, en el estudio de Delgado, Mejia y Lory (2016) se encontró que los hombres mostraban mejor actitud que las mujeres, cabe señalar este estudio se llevó a cabo en una de las universidades de este estudio. En el estudio de Rey (2019) se presentan tablas separadas para evaluar los resultados según el sexo, en donde las mujeres muestran puntuaciones más altas, sin embargo, no se menciona si esas diferencias fueron estadísticamente significativas. Por su parte, Cabrera-Enríquez et al (2013), encontraron que las mujeres tenían 17\% más probabilidades de tener buena actitud hacia la investigación que los hombres. En el estudio de Plazas, Gómez y Castro (2013) no se encontraron diferencias en la variable sexo con respecto a la actitud.

Respecto al estudio de la variable escuela en relación a la actitud hacia la investigación, Rojas (2011) encontró en su estudio con 6 universidades distintas, que no existía una diferencia significativa entre las escuelas. Ochoa-Vigo, Bello, Villanueva, Ruiz-Garay y Manrique (2016) en su estudio encontraron que los alumnos de universidades públicas tienen mejor actitud hacia la investigación que aquellos de escuelas privadas. Finalmente, el estudio de Rojas, Méndez y Rodríguez (2012) donde compararon los resultados de cinco universidades, encontraron que los estudiantes que se encontraban en universidades de la zona metropolitana tenían una mejor actitud hacia la investigación.

Con respecto al grado de avance dentro de la carrera, se encontraron estudios con conclusiones inconsistentes. En el estudio de Taraban y Logue (2012) encontraron que conforme avanzan en los años universitarios los estudiantes se distancian de la investigación y sus beneficios. Mientras que en el estudio de Acón-Hernández, Fonseca-Artavia, Artavia-Chávez, y Galán-Rodas (2015) no encontraron una relación entre el semestre y la actitud hacia la investigación.

\section{3) Actitud general hacia la investigación en estudiantes universitarios}

Los resultados de la actitud general hacia la investigación en estudiantes universitarios son igualmente inconsistentes, en donde podemos encontrar actitudes positivas hacia la investigación y negativas, dependiente el contexto del estudio.

Los resultados obtenidos en el estudio de Rey (2019) fueron que el 78,72\% tienen una alta actitud hacia la investigación, 19,15\% muy alta y 2,13\% neutra y $0 \%$ actitud baja o muy baja. En el estudio de Alonso, Alonso, y Valadez (2015) se encontró igualmente una actitud positiva; siendo el $53.2 \%$ una actitud positiva, $3.1 \%$ actitud negativa, el $6.2 \%$ actitud que tiende a ser negativa, el $34.4 \%$ tiende a ser positiva, y el $3.1 \%$ actitud muy positiva. Cabe señalar que la aplicación del instrumento fue antes de iniciar las clases de metodología e investigación en enfermería para conocer su disposición. 
En el estudio de Arellano-Sacramento, Hermoza-Moquillaza, Elías-Podestá y RamírezJulca (2017) se encontró que la mayoría de los estudiantes tenían una actitud regular hacia la investigación $(58,2 \%)$, seguido por una mala actitud $(23,2 \%)$ y finalmente una buena actitud $(18,6 \%)$. En el estudio de Rojas, Méndez y Rodríguez (2012) igualmente se muestra una tendencia hacia una actitud regular o moderada hacia la investigación $(68.4 \%)$ seguida por un índice alto (24.2\%) e índice bajo lo menos común (7.4\%).

Finalmente, con una actitud negativa se encontró el estudio de Rojas (2010) que toma en cuenta elementos institucionales, docentes y propios del estudiante, encontrando una predisposición negativa a la investigación $(42,6 \%)$, seguido por una predisposición media $(34,1 \%)$ y finalmente una buena predisposición $(23,3 \%)$.

El objetivo de esta investigación fue diseñar y validar un instrumento para evaluar la actitud hacia la investigación en estudiantes universitarios

\section{MÉTODO Y MATERIALES}

\section{Diseño de investigación}

El diseño de esta investigación fue instrumental, ya que de acuerdo a Montero y León (2002) se identifican así "todos los estudios encaminados al desarrollo de pruebas y aparatos, incluyendo tanto el diseño como la adaptación de los mismos" (p.507).

\section{Técnica o instrumento}

El diseño del instrumento se hizo a partir de la técnica de redes semánticas. El uso de esta técnica permite una aproximación a las ideas, creencias y valoraciones que tienen los estudiantes respecto a la investigación. Se utilizaron las palabras estímulo: investigación, obstáculos investigación, habilidades investigación. En las tres redes se utilizaron 5 palabras definidoras, que posteriormente se organizaron por frecuencias. En la tabla 1 se pueden observar los elementos obtenidos de las redes semánticas que fueron organizados en dos dimensiones (actitud positiva y negativa) y cuatro categorías (habilidades para la investigación, valoración positiva, obstáculos para la investigación y valoración negativa).

El cuestionario está compuesto por 28 reactivos redactados a manera de afirmación, con una escala Likert ( $0=$ muy desacuerdo; $1=$ desacuerdo; $2=$ de acuerdo; $3=$ muy de acuerdo). En la dimensión actitud negativa los reactivos tienen una puntuación inversa $(3=$ muy desacuerdo; $2=$ desacuerdo; $1=$ de acuerdo; $0=$ muy de acuerdo). Está dirigida a estudiantes universitarios, se puede aplicar de manera individual o grupal. Se responde en aproximadamente 15 minutos. 
Tabla 1.

Especificaciones del cuestionario

\begin{tabular}{|c|c|c|c|}
\hline $\begin{array}{c}\text { Dimensió } \\
\mathrm{n}\end{array}$ & Categoría & $\begin{array}{c}\text { Elementos obtenidos de } \\
\text { las redes semánticas } \\
\text { naturales }\end{array}$ & Reactivos \\
\hline \multirow{14}{*}{ 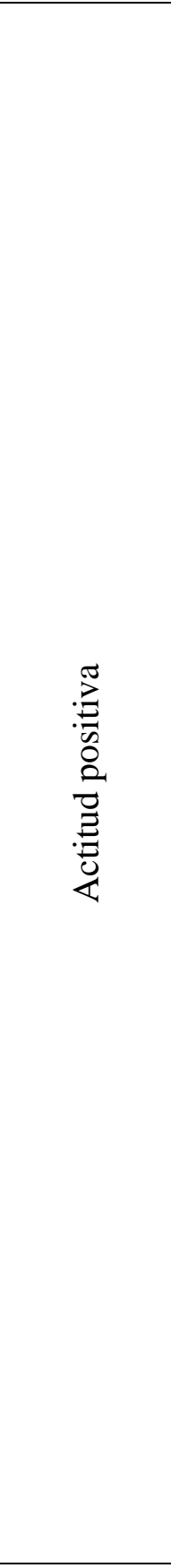 } & \multirow{7}{*}{$\begin{array}{l}\text { Habilidades } \\
\text { para la } \\
\text { investigació } \\
\text { n }\end{array}$} & \multirow{7}{*}{$\begin{array}{l}\text { trabajo en equipo }(\mathrm{f}=40), \\
\text { redacción }(\mathrm{f}=35) \text {, uso } \\
\text { APA }(\mathrm{f}=26) \text {, indagar } \\
(\mathrm{f}=26) \text {, problematizar } \\
(\mathrm{F}=24) \text {, experiencia } \\
(\mathrm{f}=24) \text {, comprensión } \\
(\mathrm{f}=22) \text {, paciencia }(\mathrm{f}=21), \\
\text { comunicar }(\mathrm{f}=17), \\
\text { inteligencia }(\mathrm{f}=15), \\
\text { tecnología }(\mathrm{f}=14), \\
\text { intervención }(\mathrm{f}=12), \\
\text { curiosidad }(\mathrm{f}=12), \text { hablar } \\
\text { en público }(\mathrm{f}=12), \text { idioma } \\
\text { ( } \mathrm{f}=8), \text { ortografía }(\mathrm{f}=8), \\
\text { organizar }(\mathrm{f}=8), \text { toma } \\
\text { decisiones }(\mathrm{f}=5), \\
\text { capacidad }(\mathrm{f}=5), \text { citar } \\
(\mathrm{f}=3) \text {, objetividad }(\mathrm{f}=3), \\
\text { trato directo }(\mathrm{f}=3), \\
\text { entender }(\mathrm{f}=3), \text { resolver } \\
(\mathrm{f}=3)\end{array}$} & $\begin{array}{l}\text { 1. Debo saber usar el formato APA para } \\
\text { hacer investigación }\end{array}$ \\
\hline & & & $\begin{array}{l}\text { 5. Debo saber trabajar en equipo para } \\
\text { hacer investigación }\end{array}$ \\
\hline & & & $\begin{array}{l}\text { 9. Debo saber redactar para hacer } \\
\text { investigación }\end{array}$ \\
\hline & & & $\begin{array}{l}\text { 13. Debo saber un segundo un idioma } \\
\text { para hacer investigación }\end{array}$ \\
\hline & & & $\begin{array}{l}\text { 17. Debo saber organizarme para hacer } \\
\text { investigación }\end{array}$ \\
\hline & & & $\begin{array}{l}\text { 21. Debo saber tomar decisiones para } \\
\text { hacer investigación }\end{array}$ \\
\hline & & & $\begin{array}{l}\text { 25. Debo saber estadística para hacer } \\
\text { investigación }\end{array}$ \\
\hline & \multirow{7}{*}{$\begin{array}{l}\text { Valoración } \\
\text { positiva }\end{array}$} & \multirow{7}{*}{$\begin{array}{l}\text { dedicación }(\mathrm{f}=29) \text {, ética } \\
(\mathrm{f}=24) \text {, creativo }(\mathrm{f}=18), \\
\text { responsable }(\mathrm{f}=9), \\
\text { seguridad }(\mathrm{f}=9), \\
\text { sensibilidad }(\mathrm{f}=8) \text {, actitud } \\
(\mathrm{f}=7), \text { motivación }(\mathrm{f}=7), \\
\text { flexible }(\mathrm{f}=4) \text {, empatía } \\
(\mathrm{f}=4) \text {, tolerante }(\mathrm{f}=4), \\
\text { confianza }(\mathrm{f}=3), \\
\text { disciplina }(\mathrm{f}=3), \text { esfuerzo } \\
(\mathrm{f}=3), \text { voluntad }(\mathrm{f}=3), \\
\text { compromiso }(\mathrm{f}=3)\end{array}$} & $\begin{array}{l}\text { 4. Hacer investigación fortalecer la ética } \\
\text { profesional }\end{array}$ \\
\hline & & & $\begin{array}{l}\text { 8. Hacer investigación fomenta la } \\
\text { creatividad }\end{array}$ \\
\hline & & & $\begin{array}{l}\text { 12. Hacer investigación desarrolla la } \\
\text { responsabilidad }\end{array}$ \\
\hline & & & $\begin{array}{l}\text { 16. Hacer investigación te vuelve más } \\
\text { comprometido }\end{array}$ \\
\hline & & & $\begin{array}{l}\text { 20. Hacer investigación desarrolla la } \\
\text { seguridad personal }\end{array}$ \\
\hline & & & $\begin{array}{l}\text { 24. Hacer investigación flexibiliza el } \\
\text { pensamiento }\end{array}$ \\
\hline & & & $\begin{array}{l}\text { 28. Hacer investigación desarrolla la } \\
\text { tolerancia a la frustración }\end{array}$ \\
\hline \multirow{4}{*}{ 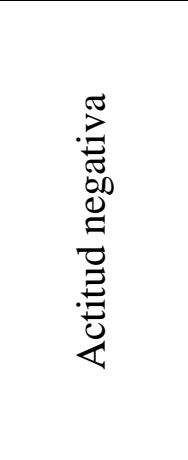 } & \multirow{4}{*}{$\begin{array}{l}\text { Obstáculos } \\
\text { para la } \\
\text { investigació } \\
\text { n }\end{array}$} & \multirow{4}{*}{$\begin{array}{l}\text { tiempo }(\mathrm{f}=77) \text {, falta de } \\
\text { dinero }(\mathrm{f}=66) \text {, falta } \\
\text { interés }(\mathrm{f}=49) \text {, falta } \\
\text { conocimiento }(\mathrm{f}=36) \text {, } \\
\text { mala organización } \\
(\mathrm{f}=22), \text { falta de } \\
\text { información }(\mathrm{f}=22) \text {, falta } \\
\text { experiencia }(\mathrm{f}=18) \text {, falta } \\
\text { de habilidades }(\mathrm{f}=11),\end{array}$} & $\begin{array}{l}\text { 2. La falta de dinero es mi mayor } \\
\text { problema para hacer investigación }\end{array}$ \\
\hline & & & $\begin{array}{l}\text { 6. La falta de tiempo es mi mayor } \\
\text { problema para hacer investigación }\end{array}$ \\
\hline & & & $\begin{array}{l}\text { 10. La falta de apoyo de mis profesores } \\
\text { es mi mayor problema para hacer } \\
\text { investigación }\end{array}$ \\
\hline & & & $\begin{array}{l}\text { 14. La falta de conocimientos es un } \\
\text { obstáculo para hacer investigación }\end{array}$ \\
\hline
\end{tabular}




\begin{tabular}{|c|c|c|}
\hline & $\begin{array}{l}\text { asesorías }(\mathrm{f}=10) \\
\text { viabilidad }(\mathrm{f}=9)\end{array}$ & $\begin{array}{l}\text { 18. La falta de apoyo de mi escuela es un } \\
\text { obstáculo para hacer investigación }\end{array}$ \\
\hline & $\begin{array}{l}\text { credibilidad }(\mathrm{f}=6) \\
\text { ignorancia }(\mathrm{f}=6)\end{array}$ & $\begin{array}{l}\text { 22. La falta de información es la razón } \\
\text { por la que no hago investigación }\end{array}$ \\
\hline & $\begin{array}{l}\text { conflictos }(\mathrm{f}=5) \text {, } \\
\text { resultados incorrectos } \\
(\mathrm{f}=5) \text {, problemas lectura } \\
(\mathrm{f}=4) \text {, plagio }(\mathrm{f}=4), \text { poco } \\
\text { contexto }(\mathrm{f}=4), \\
\text { distracciones }(\mathrm{f}=4), \\
\text { tardado }(\mathrm{f}=4), \text { malos } \\
\text { experimentos }(\mathrm{f}=4), \\
\text { disponibilidad }(\mathrm{f}=3), \\
\text { burocracia }(\mathrm{f}=3), \text { política } \\
(\mathrm{f}=3)\end{array}$ & $\begin{array}{l}\text { 26. La falta de credibilidad como } \\
\text { estudiante es la razón por la que no hago } \\
\text { investigación }\end{array}$ \\
\hline \multirow{7}{*}{$\begin{array}{l}\text { Valoración } \\
\text { negativa }\end{array}$} & \multirow{7}{*}{$\begin{array}{c}\text { desinterés }(\mathrm{f}=18) \text {, difícil } \\
(\mathrm{f}=18), \text { flojera }(\mathrm{f}=15), \\
\text { apatía }(\mathrm{f}=11) \text {, aburrido } \\
(\mathrm{f}=7) \text {, agobio }(\mathrm{f}=6), \\
\text { banalidad }(\mathrm{f}=5), \\
\text { cansancio }(\mathrm{f}=4) \text {, soberbia } \\
(\mathrm{f}=4) \text {, tedioso }(\mathrm{f}=3), \\
\text { resistencia }(\mathrm{f}=3), \\
\text { depresión }(\mathrm{f}=3), \text { estrés } \\
(\mathrm{f}=3)\end{array}$} & $\begin{array}{l}\text { 3. Me parece que hacer investigación es } \\
\text { aburrido }\end{array}$ \\
\hline & & $\begin{array}{l}\text { 7. Me parece que hacer investigación no } \\
\text { es algo interesante }\end{array}$ \\
\hline & & $\begin{array}{l}\text { 11. Me parece que la investigación no es } \\
\text { necesaria para mi profesión }\end{array}$ \\
\hline & & $\begin{array}{l}\text { 15. Me parece que la investigación es } \\
\text { difícil }\end{array}$ \\
\hline & & $\begin{array}{l}\text { 19. Me parece que hacer investigación es } \\
\text { tedioso }\end{array}$ \\
\hline & & $\begin{array}{l}\text { 23. Me parece que sólo se hace } \\
\text { investigación para la tesis }\end{array}$ \\
\hline & & $\begin{array}{l}\text { 27. Me parece que hacer investigación es } \\
\text { estresante }\end{array}$ \\
\hline
\end{tabular}

\section{Escenario}

La investigación se llevó a cabo en 3 Universidades del Estado de Morelos que ofertaban la carrera de Psicología.

La Universidad 1, es una institución pública que brinda atención a aproximadamente 900 estudiantes. Los alumnos suelen tener algún contacto con la investigación en las materias de metodología o diseño de instrumentos, algunos otros en prácticas; pero no existe un programa en concreto que promueva la investigación en estudiantes universitarios.

La Universidad 2, es una institución privada que brinda atención a aproximadamente 200 estudiantes. El sistema de la escuela es de tipo ABP (Aprendizaje Basado en Proyectos), por lo que cada año, los estudiantes tienen que desarrollar un proyecto de investigación. Sin embargo, tiene un alto nivel de rotación docente, lo que impide dar seguimiento a los proyectos, además de una estructura poca clara de asesorías. 
La Universidad 3, es una institución privada que brinda atención a aproximadamente 200 estudiantes. Los alumnos suelen tener algún contacto con la investigación en las materias de metodología o estadística, algunos otros en prácticas; pero no existe un programa en concreto que promueva la investigación en estudiantes universitarios.

\section{Participantes}

El muestreo fue no probabilístico intencional, se invitó a los estudiantes de la carrera de Psicología de las tres universidades para participar en la investigación, de manera voluntaria y en conocimiento de los objetivos de la misma. Participaron 212 estudiantes con una edad promedio de 21 años. El 32\% pertenecían al primer año, 26\% de segundo, 26\% tercer y el $16 \%$ del cuarto año de la carrera. Las características de la muestra pueden observarse en la tabla 2.

Tabla 2

Participantes por universidad.

\begin{tabular}{llcccc}
\hline \multicolumn{5}{c}{ Escuela } \\
\hline \multirow{2}{*}{ Sexo } & Mujer & Universidad 1 & Universidad 2 & Universidad 3 & Total \\
& Hombre & 46 & 37 & 66 & 149 \\
\multirow{2}{*}{ Total } & 15 & 15 & 33 & 63 \\
& & 61 & 52 & 99 & 212 \\
\hline
\end{tabular}

\section{Procedimiento}

Se solicitaron los permisos para desarrollar el proyecto en las instituciones, las cuales accedieron aclarando que era necesaria la autorización del docente que se encontrara con cada grupo. Se aplicaron las redes semánticas con las palabras estímulo: investigación, obstáculos investigación, habilidades investigación. Se aplicaron de forma anónima las redes semánticas a 120 estudiantes de la carrera de Psicología. Se obtuvieron las cargas semánticas, frecuencias y se eliminaron las palabras con un FMG, o distancia semántica, menor al $4 \%$.

Se organizaron las palabras en cuatro dimensiones: habilidades para la investigación, obstáculos para la investigación, actitud negativa hacia la investigación y actitud positiva hacia la investigación. Se crearon 7 reactivos para cada dimensión. Posteriormente se piloteó el cuestionario de 28 ítems con 95 estudiantes de las carreras de Psicología y Educación. De los resultados del pilotaje se decidió modificar la redacción de los reactivos de las dimensiones actitud negativa, ya que se obtenían correlaciones negativas en el pilotaje.

Se aplicó la versión final del cuestionario a 212 estudiantes pertenecientes de las tres Universidades, que no habían participado en el pilotaje. 


\section{Análisis de datos}

Se analizaron los datos en el programa estadístico SPSS versión 23. Se obtuvieron los datos descriptivos, Alpha de Cronbach, análisis factorial, correlación y ANOVA.

\section{RESULTADOS}

Los resultados de la presente investigación se organizan en dos apartados: 1) propiedades psicométricas del cuestionario y 2) resultados del cuestionario.

\section{1) Propiedades psicométricas}

Se obtuvieron las propiedades psicométricas del cuestionario. Para la confiabilidad se realizó una prueba de homogeneidad y se obtuvo un Alpha de Cronbach de .726, que se considera aceptable. Además, como se muestra en la tabla 3, se obtuvo el Alfa de Cronbach por reactivo, para valorar la conveniencia de suprimir algún reactivo, decidiendo dejar la estructura ya que no subía a un valor de .800 .

Tabla 3

Alfa de Cronbach si el elemento es suprimido

\begin{tabular}{|c|c|}
\hline Reactivo & $\begin{array}{c}\text { Alfa de } \\
\text { Cronbach si el } \\
\text { elemento se ha } \\
\text { suprimido }\end{array}$ \\
\hline P1 & .719 \\
\hline P2 & .733 \\
\hline P3 & .709 \\
\hline P4 & .719 \\
\hline P5 & .721 \\
\hline P6 & .719 \\
\hline P7 & .708 \\
\hline P8 & .716 \\
\hline P9 & .721 \\
\hline P10 & .718 \\
\hline P11 & .712 \\
\hline P12 & .707 \\
\hline P13 & .730 \\
\hline P14 & .732 \\
\hline P15 & .719 \\
\hline P16 & .713 \\
\hline P17 & .720 \\
\hline P18 & .724 \\
\hline P19 & .710 \\
\hline & \\
\hline
\end{tabular}




\begin{tabular}{|l|l|}
\hline P20 & .716 \\
\hline P21 & .717 \\
\hline P22 & .718 \\
\hline P23 & .717 \\
\hline P24 & .710 \\
\hline P25 & .731 \\
\hline P26 & .720 \\
\hline P27 & .709 \\
\hline P28 & .734 \\
\hline
\end{tabular}

Respecto a la validez, se obtuvo la validez de constructo con un análisis factorial con dos factores controlados con una rotación Varimax. Para verificar que la muestra contaba con las características necesarias para hacer un análisis factorial, se realizó la prueba Kaiser-Meyer-Olkin (KMO) con un puntaje de .775; mientras que la prueba de esfericidad obtuvo un puntaje de 1516. 567 con una $\mathrm{p}<.008$. Se eligieron 2 factores ya que correspondían mejor con la prueba, que tomando las categorías como factores. Ambos factores explican el 30.24\% de la varianza. En la tabla 4 pueden observarse las cargas factoriales de los reactivos.

Tabla 4

Cargas factoriales por factor

\begin{tabular}{|c|c|c|}
\hline Reactivo & Factor 1 & Factor 2 \\
\hline P16 & .710 & \\
\hline P24 & .683 & \\
\hline P21 & .663 & \\
\hline P12 & .649 & \\
\hline P20 & .638 & \\
\hline P17 & .580 & \\
\hline P9 & .512 & \\
\hline P8 & .492 & \\
\hline P4 & .488 & \\
\hline P13 & .431 & \\
\hline P28 & .424 & \\
\hline P25 & .420 & \\
\hline P1 & .412 & \\
\hline P5 & .366 & \\
\hline P19 & & .636 \\
\hline P27 & & .634 \\
\hline P3 & & .606 \\
\hline P22 & & .593 \\
\hline P15 & & .569 \\
\hline P26 & & .538 \\
\hline
\end{tabular}




\begin{tabular}{|c|c|c|} 
P23 & & .528 \\
\hline P18 & & .520 \\
\hline P10 & & .511 \\
\hline P6 & & .481 \\
\hline P11 & & .464 \\
\hline P7 & & .458 \\
\hline P14 & & .327 \\
\hline P2 & & .210 \\
\hline
\end{tabular}

\section{2) Resultados cuestionario}

Los resultados del cuestionario se organizaron en cuatro dimensiones: a) habilidades para la investigación, b) valoración positiva hacia la investigación, c) obstáculos para la investigación y d) valoración negativa hacia la investigación.

La dimensión habilidades para la investigación se muestra en la figura 1, en la que destaca que las habilidades que se consideran más importantes para hacer investigación es el uso del formato APA (89\%) y saber redactar (86\%), y organizar; mientras que la que se considera menos importante es el manejo de un segundo idioma (61\%).

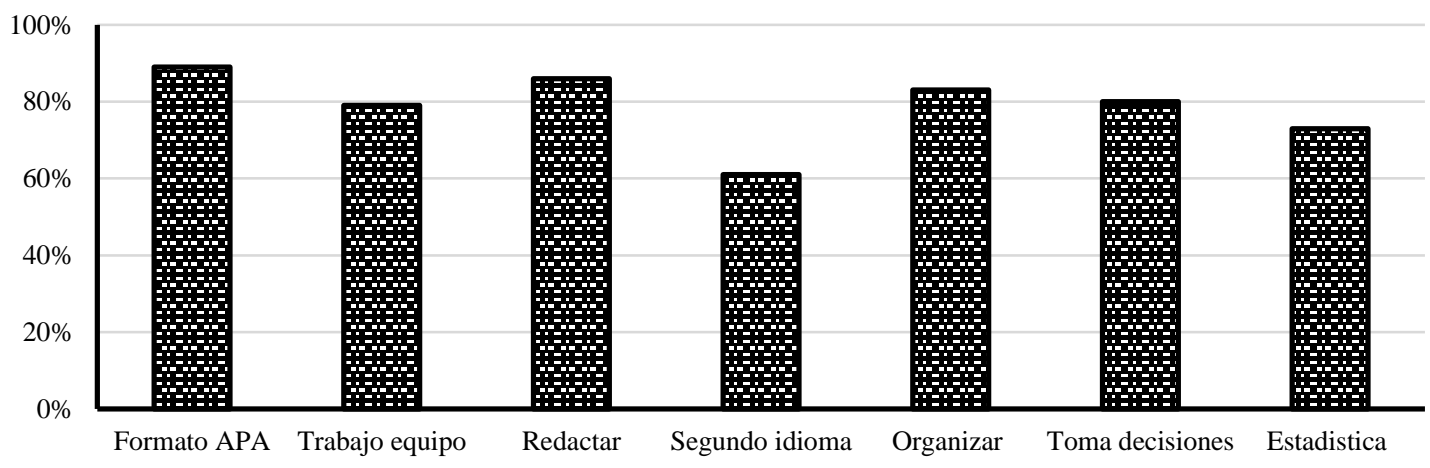

Figura 1. Habilidades para la investigación.

La figura 2 ilustra la dimensión valoración positiva hacia la investigación en la cual es notable que la investigación, a juicio de los examinados, les ayuda a ser más responsables (81\%), además de fomentar la creatividad (79\%). Mientras que consideran que en lo que menos les ayuda es a desarrollar su tolerancia a la frustración (62\%). 


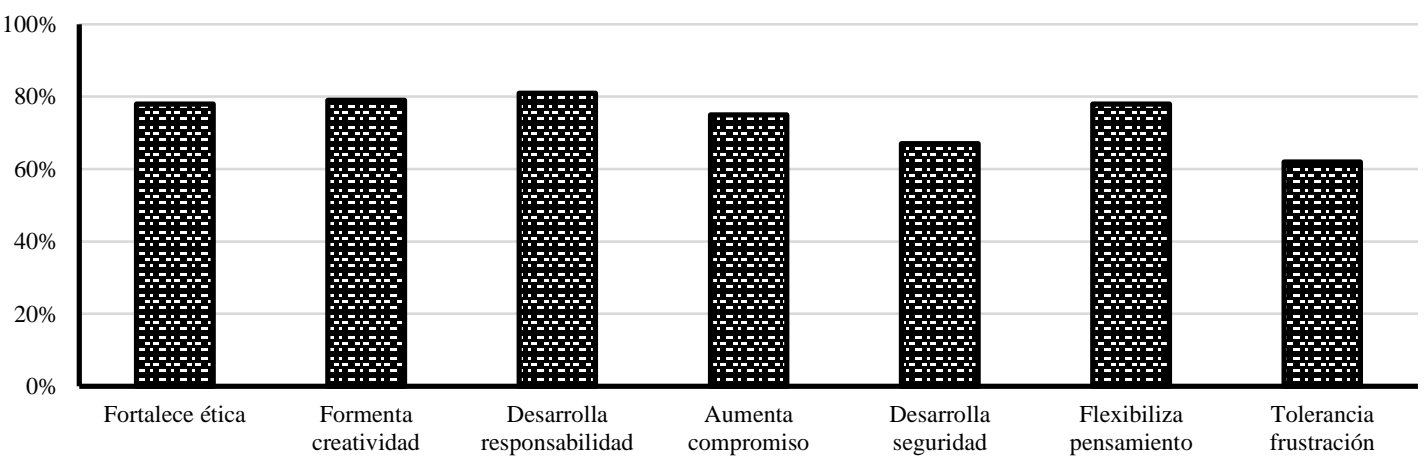

Figura 2. Valoración positiva hacia la investigación.

La dimensión obstáculos para la investigación permite identificar que los participantes consideran que la falta de conocimientos es el mayor obstáculo para hacer investigación (65\%), así como la falta de tiempo (59\%). Mientras que la falta de credibilidad (44\%) y la falta de información (49\%) son reconocidos como obstáculos menores (ver figura 3).

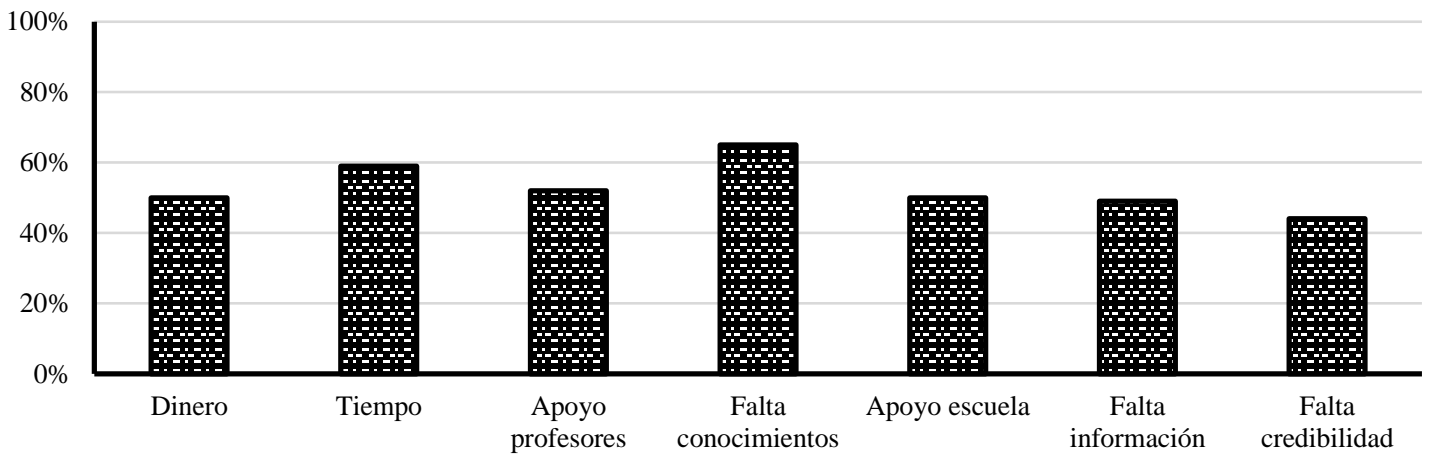

Figura 3. Obstáculos para la investigación.

En la figura 4 se aprecian los factores que fueron valorados como negativos hacia la investigación, destacando entre ellos, una percepción estresante (61\%) y tediosa (60\%), los puntajes más bajos en actitud negativa son para "Me parece que saber investigar es necesario para mi profesión" (23\%) y "Me parece que hacer investigación es algo interesante" (34\%).

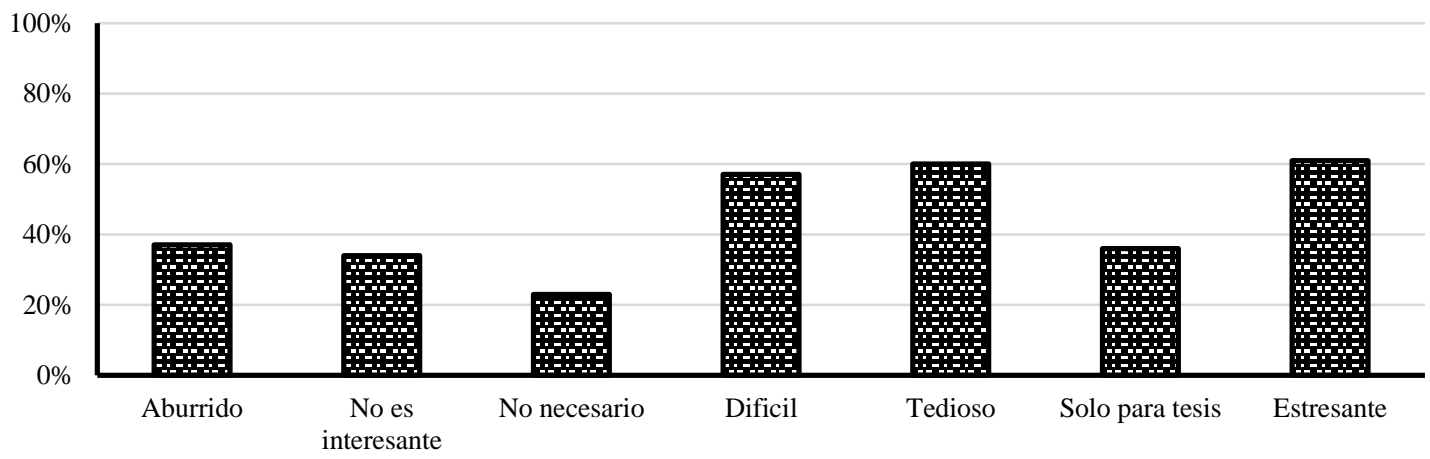

Figura 4. Valoración negativa hacia la investigación. 
Para la variable sexo no se encontraron diferencias significativas, se utilizó una U de MannWhitney debido a las $n$ desiguales, ya que en la carrera de Psicología hay más mujeres que hombres. Se obtuvo un valor de 541.500 con una $\mathrm{p}<.266$.

En cuanto a la variable Escuela, se compararon las tres universidades, no se encontraron diferencias en cuanto a la actitud hacia la investigación $(\mathrm{F}=.278, \mathrm{p}<.758)$, y con respecto a la variable grado de avance dentro de la carrera (semestre), se decidió agrupar por año, ya que la segunda universidad cuenta con un sistema de cuatrimestres, mientras que las otra dos se organizan por semestres, por lo que se agruparon por año para facilitar la comparación. Se encontró que existían diferencias en los puntajes por año de la carrera, con una $\mathrm{F}=4.013$ y una $\mathrm{p}<.008$, es decir, los estudiantes de primer año tienen mejor actitud hacia la investigación y conforme avanzan en la carrera esta disminuye (ver figura 5).

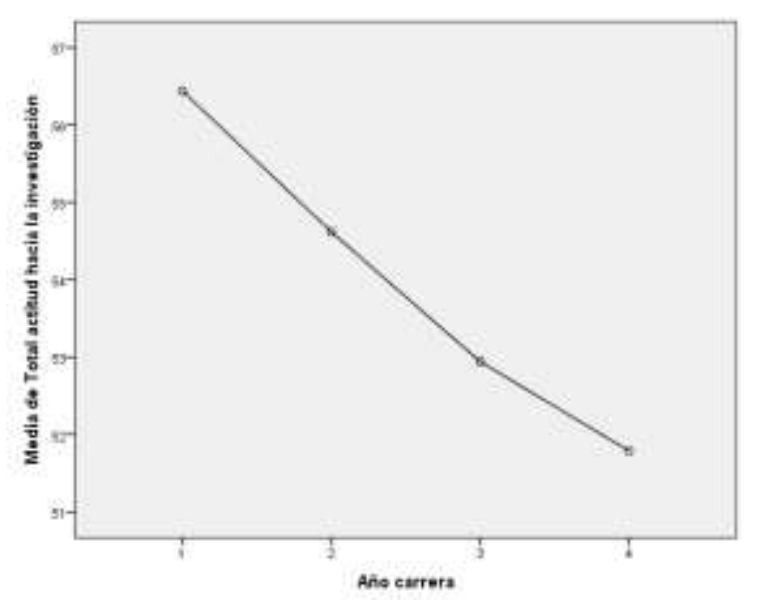

Figura 5. ANOVA por año de carrera.

En cuanto a los puntajes totales de actitud hacia la investigación se eligieron como puntos de corte los percentiles 33 y 66, para elegir los tres niveles de actitud hacia la investigación: actitud mala, regular y buena (ver tabla 5). Se encuentra que el mayor porcentaje fue para mala actitud (36.3\%), seguido por regular $(33.5 \%)$ y finalmente una actitud buena $(30.2 \%)$.

Tabla 5.

Actitud hacia la investigación

\begin{tabular}{ccc}
\hline $\begin{array}{c}\text { Actitud hacia la } \\
\text { investigación }\end{array}$ & Total & Porcentaje \\
\hline Mala & 77 & 36.3 \\
Regular & 71 & 33.5 \\
Buena & 64 & 30.2 \\
Total & 212 & 100.0 \\
\hline
\end{tabular}




\section{DISCUSIONES}

Respecto a las propiedades psicométricas del cuestionario, los valores para la confiabilidad se encontraron un Alpha de Cronbach de .726, considerándose a partir de .700 como un cuestionario con una buena consistencia interna (Quero, 2010). Aunque en la literatura se encuentran al menos dos cuestionarios publicados con un Alpha por debajo del .700 (Arellano-Sacramento, HermozaMoquillaza, Elías-Podestá \& Ramírez-Julca, 2017; Ochoa-Vigo, Bello, Villanueva, Ruiz-Garay \& Manrique, 2016). Respecto a la validez, se obtuvo mediante la validez de constructo, se organizó en dos factores que explican el 30.24\% de la varianza y que corresponden por lo descrito en la tabla de especificaciones del cuestionario. En general se encuentran propiedades psicométricas aceptables para el uso del cuestionario.

Con respecto a la dimensión positiva, se encontró que los estudiantes consideran que requieren un manejo del formato APA y habilidades de redacción, encontrándose un estudio de García, Paca, Arista, Valdez y Gómez (2018) que señala cómo estas habilidades mejoran con la investigación, pero no se encontró alguno que indague solo los requisitos que los estudiantes consideran que necesitan. Con respecto a la valoración positiva se encontró que los estudiantes consideran que la investigación desarrolla la responsabilidad, creatividad y fortalece la ética, esto coincide con los hallazgos de Barrios, Delgado y Hernández (2019), quienes compararon estudiantes con y sin experiencia en investigación.

Con respecto a la dimensión negativa, se encontró que los estudiantes consideraban que la falta de conocimientos y el tiempo eran los principales obstáculos para no hacer investigación, esto coinciden con el estudio de Bendezú, Hurtado, Medina, y Aguilar (2015). Respecto a la valoración negativa, consideran que la investigación es estresante y tediosa, coincidiendo con los resultados de Rocchi, Beaudry, Anderson, y Pelletier (2016) con jóvenes sin experiencia en investigación.

Con respecto a la variable género, los hallazgos coinciden con los de Plazas, Gómez y Castro (2013) al no encontrar diferencias significativas. La variable escuela, pese a que se esperaba que existieran diferencias con las escuelas públicas y privadas como en el estudio de Ochoa-Vigo, Bello, Villanueva, Ruiz-Garay y Manrique (2016), no se encontraron diferencias significativas, coincidiendo con el estudio de Rojas (2011). También se esperaba que la segunda universidad con el sistema de Aprendizaje Basado en Proyectos, tuvieran puntajes más altos, ya que manejan la investigación como una competencia transversal, sin embargo, la alta rotación y la falta de un modelo claro de tutorías, ha impedido que los estudiantes vivan la investigación como experiencia agradable, y por el contrario es vista como un requisito para aprobar, sin estándares de calidad. 
Finalmente, la variable semestre, se organizó por año debido a que una de las universidades maneja un sistema de cuatrimestres y las otras dos por semestres. Se encontraron resultados similares al estudio de Taraban y Logue (2012) donde conforme avanzaban en los años los estudiantes se distancian de la investigación y sus beneficios, por lo que es importante una intervención temprana en experiencias efectivas en investigación, ya que algunos estudiantes tienen su primer acercamiento hasta el desarrollo de la tesis y suelen no optar por esta forma de titulación.

Los resultados de niveles generales de actitud hacia la investigación son inconsistentes en la literatura, es decir, que dependerá en gran medida del contexto donde se desarrolle la investigación. En este estudio se encontró que el 36.3\% de los estudiantes presentaban una mala actitud hacia la investigación, coincidiendo con los hallazgos de Rojas (2010).

\section{CONCLUSIONES}

Es importante brindar experiencias de investigación a estudiantes no solo de manera temprana sino efectiva, donde puedan obtenerse los beneficios que la investigación en pregrado puede tener para este tipo de estudiantes.

En conclusión, el cuestionario de actitud hacia la investigación en estudiantes universitarios muestra propiedades psicométricas aceptables para su uso; se aconsejan futuras investigaciones en otras poblaciones para aportar evidencia de su validez en estudiantes de otros contextos.

\section{REFERENCIAS BIBLIOGRÁFICAS}

Acón-Hernández, E., Fonseca-Artavia, K., Artavia-Chávez, L., \& Galán-Rodas, E. (2015). Conocimientos y actitudes hacia la investigación científica en estudiantes de medicina de una Universidad Privada de Costa Rica, 2015. Revista del Cuerpo Médico del HNAAA, 8(4), 217-221. https://doi.org/10.35434/rcmhnaaa.2015.84.161

Acuña, D., Schmal, R., \& Klein, P. (2011). Una Plataforma Web para Gestionar los Derechos de Propiedad Intelectual Resultantes de la Investigación Universitaria. Journal of technology management \& innovation, 6(4), 258-275. https://dx.doi.org/10.4067/S071827242011000400018

Adedokun, O. A., \& Burgess, W. D. (2011). Uncovering Students' Preconceptions of Undergraduate Research Experiences. Journal of STEM Education: Innovations \& Research, 12.

Adedokun, O. A., Zhang, D., Parker, L. C., Bessenbacher, A., Childress, A., \& Burgess, W. D. (2012). Understanding how undergraduate research experiences influence student 
aspirations for research careers and graduate education. Journal of College Science Teaching, 42(1), 82-90.

Aldana de Becerra, G. M., Caraballo Martínez, G. J., \& Babativa Novoa, D. A. (2016). Escala para medir actitudes hacia la investigación (eacin): validación de contenido y confiabilidad. Aletheia, $8(2)$,

104-121. https://aletheia.cinde.org.co/index.php/ALETHEIA/article/view/325

Alonso, J., Alonso, A., \& Valadez, D. (2015). Actitud hacia la investigación científica de estudiantes de enfermería. Revista Cuidarte, 4(7), 22-35. http://dx.doi.org/10.22201/fesi.23958979e.2015.4.7.69106

Álvarez-Rebolledo, A. M., Santos, M. G., \& Barrios, E.E. (2019). Propiedades psicométricas del cuestionario" Percepción de la inclusión educativa en nivel superior". Sinéctica, (53) 1-21. http://dx.doi.org/10.31391/S2007-7033(2019)0053-009

Arellano-Sacramento, C., Hermoza-Moquillaza, R. V., Elías-Podestá, M., \& Ramírez-Julca, M. (2017). Actitud hacia la investigación de estudiantes universitarios en Lima, Perú. FEM: Revista de la Fundación Educación Médica, 20(4), 191-197. http://scielo.isciii.es/scielo.php?script=sci_arttext\&pid=S2014-

$98322017000400006 \& \operatorname{lng}=\mathrm{es} \& \mathrm{nrm}=$ iso

Barrios, E., E., Delgado, U., \& Hernández, E. (2019). Diferencias cualitativas entre formación investigativa e investigación formativa de estudiantes universitarios. Revista Digital de Investigación en Docencia Universitaria, $\quad 13(1), \quad 68-85$. https://doi.org/10.19083/ridu.2019.735

Bendezú, G., Hurtado, S., Medina, C. E., \& Aguilar, P. (2015). Apreciación sobre capacitación en investigación y publicación científica en estudiantes universitarios. Investigación en educación médica, 4(13), 50-51. http://www.scielo.org.mx/scielo.php?script=sci_arttext\&pid=S2007$50572015000100009 \& \operatorname{lng}=\mathrm{es} \& \mathrm{nrm}=$ iso

Bonner, A. N. N., \& Sando, J. (2008). Examining the knowledge, attitude and use of research by nurses. Journal of Nursing Management, 16(3), 334-343. https://doi.org/10.1111/j.13652834.2007.00808.x

Cabrera-Enríquez, J. A., Cruzado-Mendoza, C., Purizaca-Rosillo, N., López-Samanamú, R. O., Lajo-Aurazo, Y., Peña-Sánchez, E. R., Apolaya-Segura, M., \& Díaz-Vélez, C. (2013). Factores asociados con el nivel de conocimientos y la actitud hacia la investigación en estudiantes de medicina en Perú, 2011. Revista Panamericana de Salud Pública, 33, 166173. https://www.scielosp.org/article/rpsp/2013.v33n3/166-173/ 
Canales, A. (2011). El dilema de la investigación universitaria. Perfiles educativos, 33(spe), 3444. Recuperado en 11 de abril de 2020, de http://www.scielo.org.mx/scielo.php?script=sci_arttext\&pid=S0185-

\section{4\&lng=es\&tlng=es}

Castro, S. P. (2017). Diseño y validación de un instrumento para evaluar la actitud hacia la investigación formativa en estudiantes universitarios. Actualidades Pedagógicas, 1(70), 165-182. https://doi.org/10.19052/ap.3996

Castro, Y., Sihuay-Torres, K., \& Perez-Jiménez, V. (2018). Producción científica y percepción de la investigación por estudiantes de odontología. Educación Médica, 19(1), 19-22. https://doi.org/10.1016/j.edumed.2016.11.001

Chara-Saavedra, P., \& Olortegui-Luna, A. (2018). Factores asociados a la actitud hacia la investigación en estudiantes universitarios de enfermería. CASUS. Revista De Investigación y Casos en Salud, 3(2), 83-88. https://doi.org/10.35626/casus.2.2018.73

Criollo, M., Romero, M., \& Fontaines-Ruiz, T. (2017). Autoeficacia para el aprendizaje de la investigación en estudiantes universitarios. Psicología Educativa, 23(1), 63-72. http://dx.doi.org/10.1016/j.pse.2016.09.002

Delgado, U., Mejia J. C., y Lory, C. L. (2016) Análisis de actitud y motivación hacia la ciencia. Revista Vertientes, 16(2). 160-161.

Escalante, E., Repetto, A. M., \& Mattinello, G. (2012). Exploración y análisis de la actitud hacia la estadística en alumnos de psicología. Liberabit, 18(1), 15-26. http://www.scielo.org.pe/scielo.php?script=sci_arttext\&pid=S1729-

$48272012000100003 \& \operatorname{lng}=\mathrm{es} \& \mathrm{nrm}=$ iso

García, N. M., Paca, N. K., Arista, S. M, Valdez, B. B., \& Gómez, I. I. (2018). Investigación formativa en el desarrollo de habilidades comunicativas e investigativas. Revista de Investigaciones Altoandinas, 20(1), 125-136. https://dx.doi.org/10.18271/ria.2018.336

Linn, M. C., Palmer, E., Baranger, A., Gerard, E., \& Stone, E. (2015). Undergraduate research experiences: Impacts and opportunities. Science, 347(6222), 1261757. http://dx.doi.org/10.1126/science.1261757

Mamani, O. J. (2015). Actitud hacia la investigación y su importancia en la elección de la modalidad de tesis para optar el título profesional. Revista Científica de Ciencias de la Salud, 4(1), 22-27.

Mihi-Ramírez, A., Melchor Ferrer, E., Arteaga-Ortiz, J., \& Ojeda, S. (2017). Adquisición de competencias para evitar el plagio académico en la Enseñanza Superior. https://sudocument.ulpgc.es/handle/10553/25389 
Montero, I., \& León, O. G. (2002). Clasificación y descripción de las metodologías de investigación en Psicología. International journal of clinical and health psychology, 2(3), 503-508. http://www.aepc.es/ijchp/articulos_pdf/ijchp-53.pdf

Ochoa-Vigo, K., Bello, C., Villanueva, M. E., Ruiz-Garay, M. I., \& Manrique, G. A. (2016). Percepción y actitud del universitario de enfermería sobre su formación en investigación. Revista Médica Herediana, 27(4), 204-215. http://dx.doi.org/10.20453/rmh.v27i4.2989

Ortega, R. J., Veloso, R.D., \& Hansen, O. S. (2018). Percepción y actitudes hacia la investigación científica. Academo, 5(2), 101-109. http://dx.doi.org/10.30545/academo.2018.jul-dic.2

Pérez, L. E., Aparicio, A. S., Bazán, J. L., \& Abdounur, O. J. (2015). Actitudes hacia la estadística de estudiantes universitarios de Colombia. Educación matemática, 27(3), 111-149. http://www.scielo.org.mx/scielo.php?script=sci_arttext\&pid=S1665-

$58262015000300111 \& \operatorname{lng}=\mathrm{es} \& \operatorname{lng}=\mathrm{es}$

Plazas, M., Gómez, M., \& Castro, C. A. (2013). Actitud de estudiantes de Ciencias de la Salud hacia el conocimiento científico. Revista Ciencias de la Salud, 11(1), 83-91. https://www.redalyc.org/articulo.oa?id=562/56226330008

Quero, M. (2010). Confiabilidad y coeficiente Alpha de Cronbach. Telos, 12(2), 248-252. https://www.redalyc.org/articulo.oa?id=993/99315569010

Rey, M. R. M. (2019). Actitudes hacia la investigación en los estudiantes de la carrera de Medicina Humana de la Universidad Peruana Los Andes. Educación Médica, 20, 95-98. https://doi.org/10.1016/j.edumed.2017.10.012

Rocchi, M., Beaudry, S. G., Anderson, C., \& Pelletier, L. G. (2016). The perspective of undergraduate research participant pool nonparticipants. Teaching of Psychology, 43(4), 285-293.https://doi.org/10.1177/0098628316662756

Rojas, M. (2011). La actitud estudiantil sobre la investigación en la universidad. Un estudio de caso en seis universidades de Colombia. Investigación \& Desarrollo, 18(2), 370389http://rcientificas.uninorte.edu.co/index.php/investigacion/article/viewArticle/1136

Rojas, H. M., Méndez, R., \& Rodríguez, Á. (2012). Índice de actitud hacia la investigación en estudiantes del nivel de pregrado. Entramado, 8(2), 216-229. https://www.redalyc.org/articulo.oa?id=2654/265425848014

Taraban, R., \& Logue, E. (2012). Academic factors that affect undergraduate research experiences. Journal of Educational Psychology, 104(2), 499-514. https://doi.org/10.1037/a0026851 


\section{Cuestionario de Actitud hacia la Investigación}

(Barrios y Delgado, 2020)

Instrucciones. A continuación, se presentan una serie de sentencias con respecto a la investigación, y a la derecha de cada una, cuatro espacios que representan el grado de acuerdodesacuerdo con lo que indica cada afirmación. Marca con una X la que creas que represente mejor lo que piensas. Recuerda que no existe una respuesta correcta o incorrecta.

\begin{tabular}{|c|c|c|c|c|c|}
\hline & & 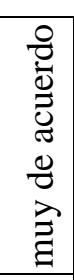 & 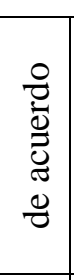 & 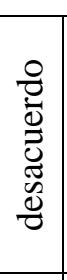 & 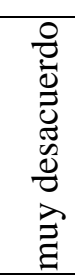 \\
\hline 1 & Debo saber usar el formato APA para hacer investigación & & & & \\
\hline 2 & La falta de dinero es mi mayor problema para hacer investigación & & & & \\
\hline 3 & Me parece que hacer investigación es aburrido & & & & \\
\hline 4 & Hacer investigación fortalecer la ética profesional & & & & \\
\hline 5 & Debo saber trabajar en equipo para hacer investigación & & & & \\
\hline 6 & La falta de tiempo es mi mayor problema para hacer investigación & & & & \\
\hline 7 & Me parece que hacer investigación no es algo interesante & & & & \\
\hline 8 & Hacer investigación fomenta la creatividad & & & & \\
\hline 9 & Debo saber redactar para hacer investigación & & & & \\
\hline 10 & $\begin{array}{l}\text { La falta de apoyo de mis profesores es un problema para hacer } \\
\text { investigación }\end{array}$ & & & & \\
\hline 11 & Me parece que la investigación no es necesaria para mi profesión & & & & \\
\hline 12 & Hacer investigación desarrolla la responsabilidad & & & & \\
\hline 13 & Debo saber un segundo un idioma para hacer investigación & & & & \\
\hline 14 & La falta de conocimientos es un obstáculo para hacer investigación & & & & \\
\hline 15 & Me parece que la investigación es difícil & & & & \\
\hline 16 & Hacer investigación te vuelve más comprometido & & & & \\
\hline 17 & Debo saber organizarme para hacer investigación & & & & \\
\hline 18 & $\begin{array}{l}\text { La falta de apoyo de mi escuela es un obstáculo para hacer } \\
\text { investigación }\end{array}$ & & & & \\
\hline 19 & Me parece que hacer investigación es tedioso & & & & \\
\hline
\end{tabular}




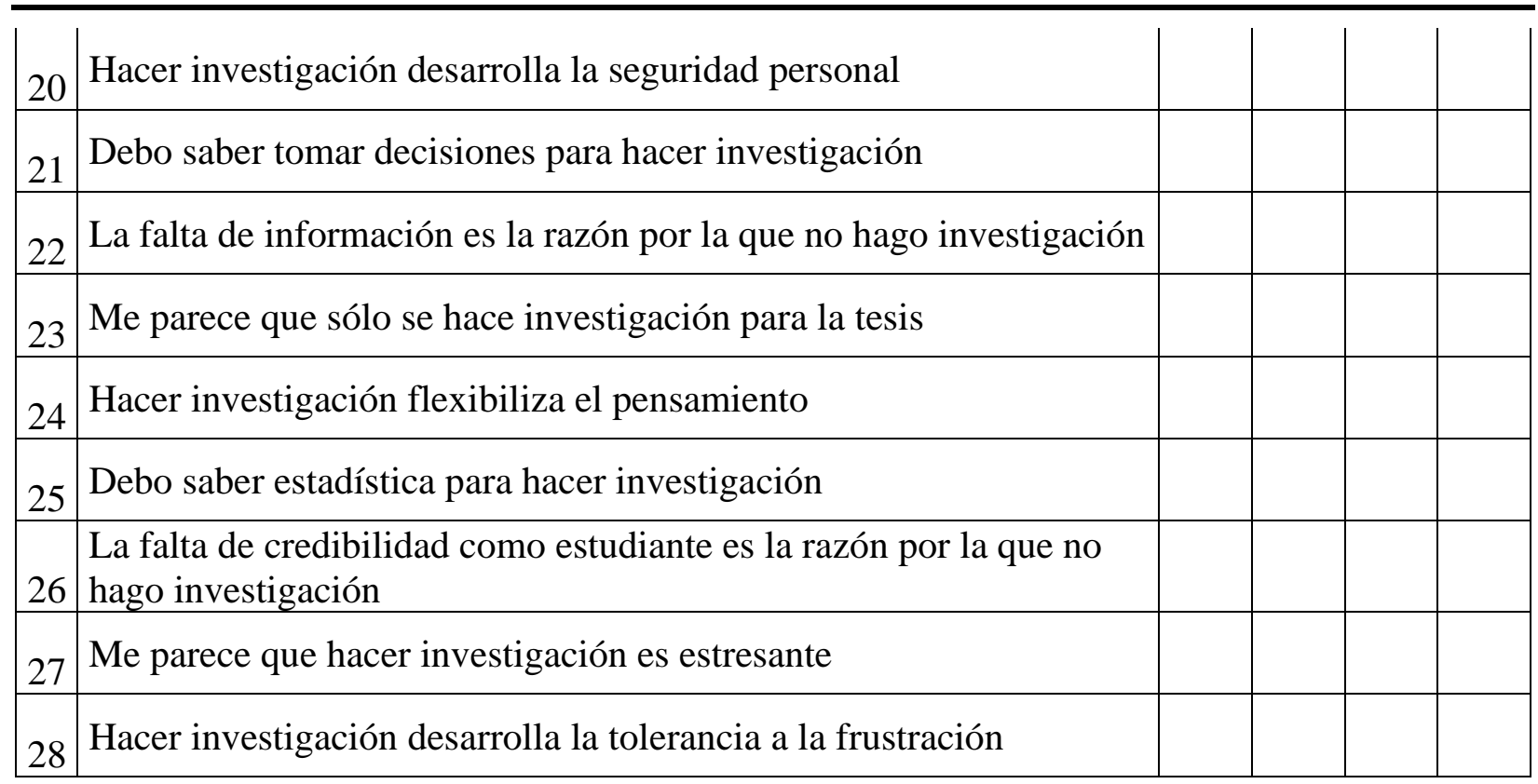

\section{Gracias por tu participación}

\section{Cuestionario de actitud hacia la investigación}

El cuestionario de actitud hacia la investigación está dirigido a estudiantes de nivel universitario. Su aplicación puede ser individual o grupal. Se aplica en un tiempo aproximado de 15 minutos.

El objetivo es evaluar las actitudes, creencias, ideas, y prejuicios, que tienen los estudiantes con respecto a la investigación. El cuestionario está compuesto por dos dimensiones (actitud positiva y actitud negativa) y cuatro componentes (habilidades para la investigación, valoración positiva, obstáculos para la investigación y valoración negativa). La distribución de 7 reactivos por categoría permite trabajar con frecuencias y puntuaciones directas.

\begin{tabular}{|c|c|c|}
\hline Dimensión & Categoría & Reactivos \\
\hline \multirow{2}{*}{ Actitud positiva } & Habilidades para la investigación & $1,5,9,13,17,21,25$ \\
\cline { 2 - 3 } & Valoración positiva & $4,8,12,16,20,24,28$ \\
\hline \multirow{2}{*}{ Actitud negativa } & Obstáculos para la investigación & $2,6,10,14,18,22,26$ \\
\cline { 2 - 3 } & Valoración negativa & $3,7,11,15,19,23,27$ \\
\hline
\end{tabular}

\section{Evaluación}

Si se desea graficar las frecuencias se deberá trabajar con las puntuaciones directas y convertirlas a porcentajes. Sin embargo, para evaluar los niveles de actitud se deberán corregir las puntuaciones de la dimensión de actitud negativa. Para calificar el cuestionario, los reactivos correspondientes a las categorías habilidades para la investigación y valoración positiva, se puntúan de la siguiente forma:

\begin{tabular}{|c|c|c|c|}
\hline Muy de acuerdo & De acuerdo & Desacuerdo & Muy desacuerdo \\
\hline 3 & 2 & 1 & 0 \\
\hline
\end{tabular}


Los reactivos correspondientes a las categorías de obstáculos para la investigación y valoración negativa puntúan de manera inversa, quedando de la siguiente manera:

\begin{tabular}{|c|c|c|c|}
\hline Muy de acuerdo & De acuerdo & Desacuerdo & Muy desacuerdo \\
\hline 0 & 1 & 2 & 3 \\
\hline
\end{tabular}

Para determinar los 3 niveles de actitud hacia la investigación (buena, regular, mala) se recomiendan como puntos de corte los percentiles 33 y 66 .

\section{Conflicto de intereses / Competing interests:}

Los autores declaran que no incurre en conflictos de intereses.

\section{Rol de los autores / Authors Roles:}

Erika Barrios: conceptualización, curación de datos, análisis formal, adquisición de fondos, investigación, metodología, administración del proyecto, recursos, software, supervisión, validación, visualización, escritura preparación del borrador original, escritura - revisar \& amp; edición.

Ulises Delgado: conceptualización, investigación, metodología, administración del proyecto, recursos, software, supervisión, validación, visualización, escritura - preparación del borrador original, escritura - revisar \& amp; edición.

\section{Fuentes de financiamiento / Funding:}

Los autores declaran que no recibieron un fondo específico para esta investigación.

\section{Aspectos éticos / legales; Ethics / legals:}

Los autores declaran no haber incurrido en aspectos antiéticos, ni haber omitido aspectos legales en la realización de la investigación. 